\title{
Development and characterization of nickel-zinc spinel ferrite for microwave absorption at $2.4 \mathrm{GHz}$
}

\author{
A KAKIRDE, B SINHA and S N SINHA* \\ Department of Electronics and Computer Engineering, Indian Institute of Technology, Roorkee 247 667, India
}

MS received 5 January 2008; revised 18 April 2008

\begin{abstract}
This paper deals with the development and characterization of nickel-zinc spinel ferrite $\left(\mathrm{Ni}_{(1-\delta)}\right.$ $\mathrm{Zn}_{\delta} \mathrm{Fe}_{2} \mathrm{O}_{4}$ ) for microwave absorption at $2.4 \mathrm{GHz}$ (ISM band). The ferrite powder was prepared by dry attrition and sintering process. Complex permittivity and permeability of the prepared sample have been determined by measuring its scattering parameters with the help of a vector network analyser. The measured parameters have been used to determine its wave absorption properties over a frequency range $2 \cdot 1-2 \cdot 6 \mathrm{GHz}$.
\end{abstract}

Keywords. Ferrites; microwave absorbers; complex permeability.

\section{Introduction}

Microwave absorbers are very useful in many applications like radar cross-section reduction, EMI/EMC problems, etc. Moreover, with the recent rapid increase in the use of portable telephones, cell phones and other wireless devices, public concerns regarding potential health hazards due to the electromagnetic energy emitted by these devices have been growing. So, it is desirable that the level of RF power absorbed in the human body be restricted to a safe limit (Jung and Lee 2002). This can be achieved by using microwave absorbers in the form of shields attached to the cell phones and wireless devices. There are a number of wireless devices that operate in the ISM band (around $2.4 \mathrm{GHz}$ ). This necessitates the development of microwave absorbers that operate in the ISM band. There are various types of microwave absorbers, viz. dielectric absorbers, magnetic absorbers, resonant absorbers, and hybrid absorbers. Ferrites are preferred as microwave absorbers for portable wireless devices operating in the ISM band because they are compact, light weight, less costly and can be easily developed (Wang et al 1999). In view of its several potential applications, a nickel-zinc spinel ferrite microwave absorber has been developed and characterized for use in the $2.4 \mathrm{GHz}$ ISM band.

\section{Preparation of ferrite}

Nickel-zinc spinel ferrite $\left(\mathrm{Ni}_{(1-\delta)} \mathrm{Zn}_{\delta} \mathrm{Fe}_{2} \mathrm{O}_{4}\right)$ for $\delta=0.595$ was prepared by dry attrition and sintering process. Raw materials used in the preparation of nickel-zinc spinel ferrite are nickel carbonate $\left(\mathrm{NiCO}_{3}\right)$, zinc oxide $(\mathrm{ZnO})$ and

*Author for correspondence (sn_sinha@ieee.org) ferric oxide $\left(\mathrm{Fe}_{2} \mathrm{O}_{3}\right)$. The procedure for preparation of ferrite is described by the flowchart in figure 1 (Meshram et al 2002). The raw materials were generally in the form of oxides and carbonates. These were first dried in an oven for $3-4 \mathrm{~h}$ at a temperature of $110^{\circ} \mathrm{C}$ to remove moisture. The dried constituents were then mixed by weight in accordance with the chemical formula and then grinded in a ball-milling machine for about $48 \mathrm{~h}$ to obtain a well mixed powder having a particle size of $<100 \mu \mathrm{m}$. The mixture was then sintered for about $8 \mathrm{~h}$ under ambient atmosphere and heating rate of $20^{\circ} \mathrm{C} / \mathrm{mt}$ to a temperature of $1150^{\circ} \mathrm{C}$. Thereafter, it was crushed again in a ballmilling machine to achieve a particle size of 5-10 microns.

The chemical formula for $\mathrm{Ni}-\mathrm{Zn}$ spinel ferrite is $\mathrm{Ni}_{(1-\delta)}$ $\mathrm{Zn}_{\delta} \mathrm{Fe}_{2} \mathrm{O}_{4}$, where $\delta$ is the fractional atomic weight. The chemical reaction involved in the preparation of $\mathrm{Ni}-\mathrm{Zn}$ spinel ferrite is described by the equation

$$
\begin{aligned}
& \quad(1-\delta) \mathrm{NiCO}_{3}+\delta \mathrm{ZnO}+\mathrm{Fe}_{2} \mathrm{O}_{3} \rightarrow \\
& \qquad \mathrm{Ni}_{(1-\delta)} \mathrm{Zn}_{\delta} \mathrm{Fe}_{2} \mathrm{O}_{4}+(1-\delta) \mathrm{CO}_{2} .
\end{aligned}
$$

The ferrimagnetic resonance frequency, $f_{\mathrm{r}}$, of the ferrite depends on $\delta$, which decides the chemical composition of the ferrite (Meshram et al 2003). In order to find the required value of $\delta$ for achieving ferrimagnetic resonance at the desired frequency of $2.4 \mathrm{GHz}$, an empirical relation between $\delta$ and $f_{\mathrm{r}}$ has been developed. For this purpose, three samples of $\mathrm{Ni}-\mathrm{Zn}$ spinel ferrite were prepared with $\delta=0.3,0.5$ and 0.7 . The absorption coefficient for each sample was experimentally measured using a HP-8720B Network Analyzer. The experimental setup is shown in figure 2 .

The length of each sample was taken to be $1.8 \mathrm{~cm}$. The samples were held in a co-axial sample holder consisting of a $50-\Omega$ airline shown in figure 3 and the scattering 
parameters were measured. The absorption coefficient, $A$, was then calculated as

$$
A=1-\left|S_{11}\right|^{2}-\left|S_{21}\right|^{2} \text {. }
$$

Equation (2) represents the law of power conservation. The first term of the right-hand side is the incident power, the second term is the power reflected by the ferrite sample to port-1, and the third term is the power transmitted through the sample and delivered at port-2.

The ferrimagnetic resonance frequency, $f_{\mathrm{r}}$, was taken as the frequency at which the absorption coefficient was maximum. The values of $\delta$ and corresponding values of $f_{\mathrm{r}}$ for the three samples are given in table 1 .

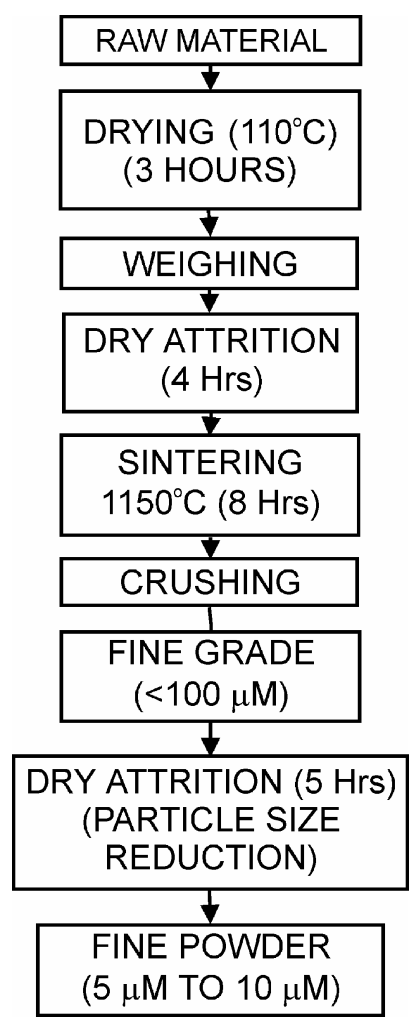

Figure 1. Preparation of $\mathrm{Ni}-\mathrm{Zn}$ ferrite.

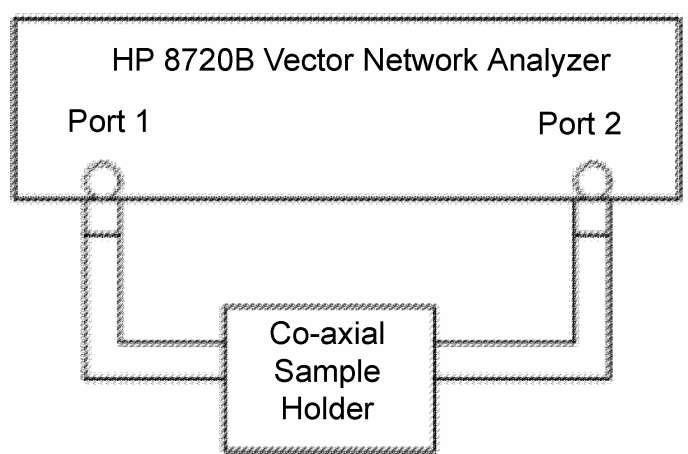

Figure 2. Setup for finding the empirical relation between $\delta$ and $f_{\mathrm{r}}$.
A linear empirical relation between $\delta$ and $f_{\mathrm{r}}$ was derived from the data in table 1 , by curve fitting as depicted in figure 4 , and is given by

$$
\delta=1.926905 f_{\mathrm{r}}-4 \cdot 02907 .
$$

Substituting $f_{\mathrm{r}}=2.4 \mathrm{GHz}$ in the empirical relation yields $\delta=0.595$.

The nickel-zinc spinel ferrite was developed for $\delta=0.595$ to give maximum absorption of microwave power at $2 \cdot 4 \mathrm{GHz}$.

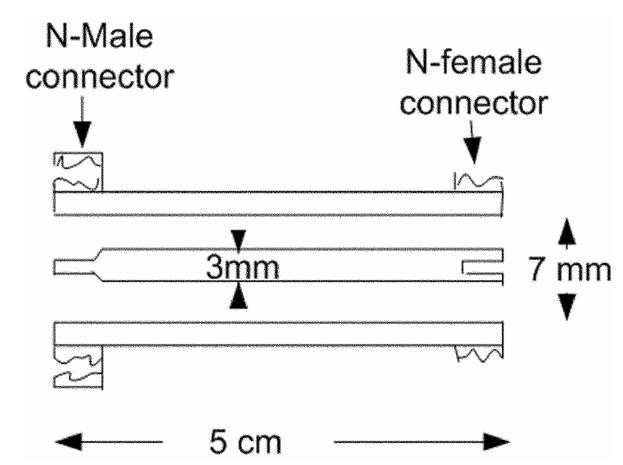

Figure 3. A coaxial line as sample holder.

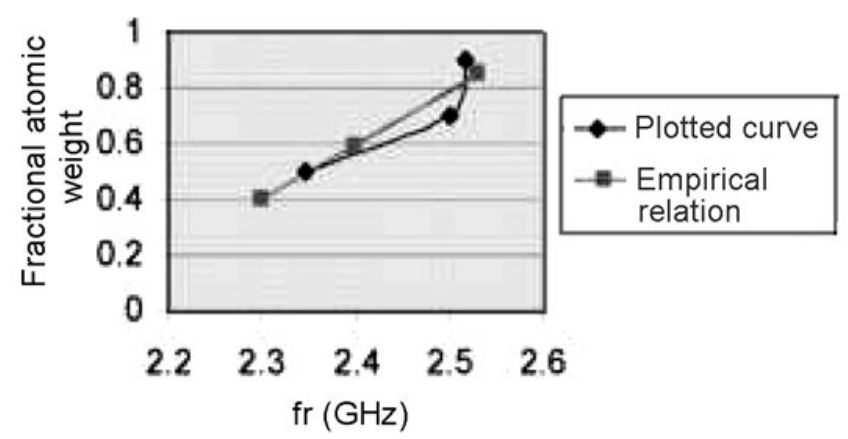

Figure 4. Empirical relation between $\delta$ and $f_{\mathrm{r}}$.

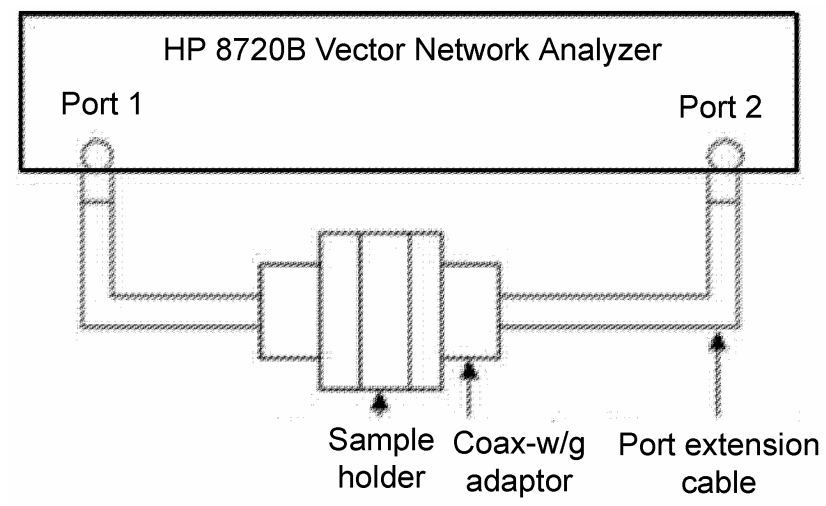

Figure 5. Setup for measuring scattering parameters of ferrite sample. 

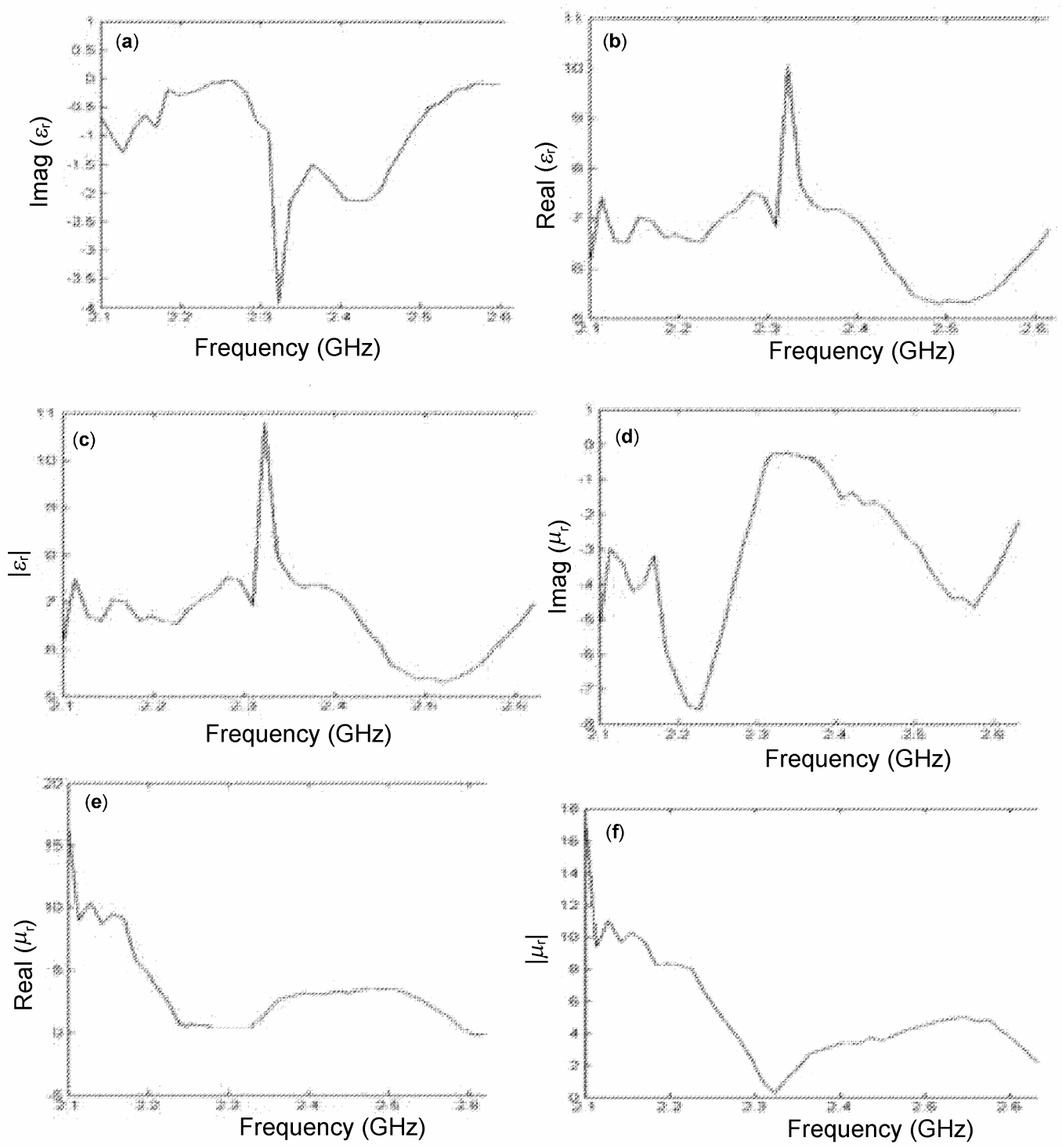

Figure 6. (a) Imaginary $\left(\varepsilon_{\mathrm{r}}\right)$ vs frequency, (b) real $\left(\varepsilon_{\mathrm{r}}\right)$ vs frequency, (c) $\left|\varepsilon_{\mathrm{T}}\right|$ vs frequency, (d) imaginary $\left(\mu_{\mathrm{r}}\right)$ vs frequency, $(\mathbf{e})$ real $\left(\mu_{\mathrm{r}}\right)$ vs frequency and (f) $\left|\mu_{\mathrm{r}}\right|$ vs frequency.

Table 1. Ferrimagnetic resonance frequency for various values of $\delta$.

\begin{tabular}{ll}
\hline$\delta$ & Measured $f_{\mathrm{r}}(\mathrm{GHz})$ \\
\hline $0 \cdot 5$ & $2 \cdot 346$ \\
$0 \cdot 7$ & $2 \cdot 5$ \\
$0 \cdot 9$ & $2 \cdot 517$ \\
\hline
\end{tabular}

3. Measurement of complex relative permittivity, permeability and absorption coefficient of developed ferrite

The complex relative permittivity, permeability and absorption coefficient of the developed ferrite were calculated from the scattering parameters of its sample. The ferrite sample was prepared in the form of a sheet of
$1 \mathrm{~mm}$ thickness and it was held in a S-band waveguide flange. The HP-8720B Network Analyzer was used to measure its scattering parameters using the experimental setup shown in figure 5 .

Scattering parameters were measured over a frequency range of $2 \cdot 1-2 \cdot 6 \mathrm{GHz}$ and the complex relative permittivity and permeability were calculated using the method proposed by Weir (1974), which can be summarized as follows:

The propagation factor for a wave propagating through a dielectric medium is defined as

$$
P=e^{-\gamma d}=e^{-(\alpha+j \beta) d},
$$

where $\gamma, \alpha$ and $\beta$ are, respectively the propagation constant, the attenuation constant and the phase-shift constant of the wave and $d$ the thickness of the medium. 


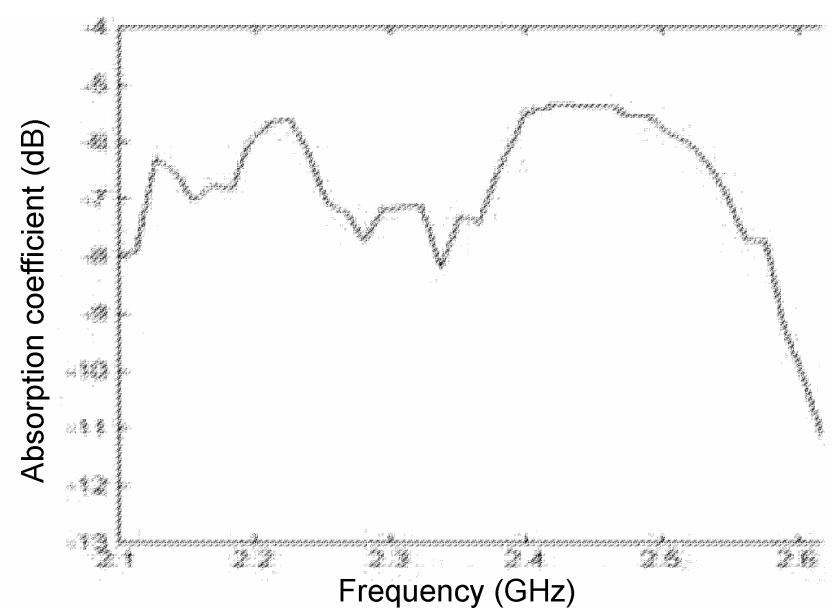

Figure 7. Absorption coefficient $(\mathrm{d} B)$ vs frequency.

The reflection coefficient, $\Gamma$, at the interface of the airfilled and dielectric-filled transmission lines can be found from the scattering parameters for a sample of finite length, $d$, as

$$
\Gamma=\chi \pm \sqrt{\chi^{2}-1}
$$

where $\chi=\frac{S_{11}^{2}-S_{21}^{2}+1}{2 S_{11}}$.

The propagation factor, $P$, can now be found from

$$
P=\frac{S_{11}+S_{21}-\Gamma}{1-\left(S_{11}+S_{21}\right) \Gamma} \text {. }
$$

The complex permittivity and permeability can be determined from $P$ and $\Gamma$ as

$$
\begin{aligned}
& \frac{1}{U^{2}}=\left(\frac{\varepsilon_{\mathrm{r}} \mu_{\mathrm{r}}}{\lambda_{0}^{2}}-\frac{1}{\lambda_{\mathrm{c}}^{2}}\right)=-\left[\frac{1}{2 \pi d} \ln \left(\frac{1}{P}\right)\right]^{2}, \\
& \mu_{\mathrm{r}}=\frac{1+\Gamma}{U(1-\Gamma) \sqrt{\frac{1}{\lambda_{0}^{2}}-\frac{1}{\lambda_{\mathrm{c}}^{2}}}}, \\
& \varepsilon_{\mathrm{r}}=\frac{\lambda_{0}^{2}}{\mu_{\mathrm{r}}}\left[\frac{1}{U^{2}}+\frac{1}{\lambda_{\mathrm{c}}^{2}}\right],
\end{aligned}
$$

where $\lambda_{0}$ and $\lambda_{\mathrm{c}}$ are, respectively the free space and cutoff wavelengths and

$$
\operatorname{Re}\left(\frac{1}{U}\right)=\frac{1}{\lambda_{\mathrm{g}}} .
$$

Variations in the real and imaginary parts of complex relative permittivity and permeability have been computed over the frequency range $2 \cdot 1-2 \cdot 6 \mathrm{GHz}$ and are shown in figures 6(a)-(f).

From figure 6(a), it is seen that the imaginary part of $\varepsilon_{\mathrm{r}}$ has its maximum value at a frequency of around $2 \cdot 32 \mathrm{GHz}$. At this frequency the imaginary part of $\mu_{\mathrm{r}}$ is relatively small (figure 6(d)). Thus, the losses are predominantly dielectric at this frequency. However, at the desired frequency of $2.4 \mathrm{GHz}$, the imaginary parts of both $\varepsilon_{\mathrm{r}}$ and $\mu_{\mathrm{r}}$ are in the range of $1 \cdot 8-2 \cdot 0$. Thus, at this frequency the material has both the dielectric losses as well as magnetic losses.

The absorption coefficient is calculated using (2) and is plotted over a frequency range of $2 \cdot 1-2 \cdot 6 \mathrm{GHz}$, as shown in figure 7 .

It can be seen that the maximum absorption having an average value of $7.5 \mathrm{~dB} / \mathrm{mm}$ occurs in the frequency range of $2.3-2.38 \mathrm{GHz}$. At the desired frequency of $2.4 \mathrm{GHz}$, the material exhibits an absorption of around $6 \mathrm{~dB} / \mathrm{mm}$ and is due both to the dielectric losses and magnetic losses in the ferrite.

\section{Conclusions}

An empirical relation between $f_{\mathrm{r}}$ and $\delta$ has been derived using three samples of $\mathrm{Ni}-\mathrm{Zn}$ spinel ferrite with known compositions. Based on this, ferrite sample at $2.4 \mathrm{GHz}$ was prepared and tested. It was found that while the peak absorption occurs in the frequency range of $2 \cdot 3-2 \cdot 38 \mathrm{GHz}$, which is slightly lower than the desired frequency of $2.4 \mathrm{GHz}$, reasonably large absorption of about $6 \mathrm{~dB} / \mathrm{mm}$ has been achieved at the desired frequency. Improved results can be obtained by using more samples for the determination of $\delta$ and controlling its value more accurately in the ferrite preparation process.

\section{Acknowledgement}

This work was carried out with financial support from the Department of Information Technology, Government of India.

\section{References}

Jung Minseok and Lee Bomson 2002 Proceedings of IEEE APS international symposium, Vol. 1, p. 444

Meshram M R et al 2002 Proceedings of IEEE APS international symposium, Vol. 2, p. 790

Meshram M R et al 2003 Microw. Opt. Technol. Lett. 36352

Wang Jianquing et al 1999 Proceedings of IEEE international symposium on electromagnetic compatibility, Vol. 2, p. 822

Weir William B 1974 Proc. IEEE 6233 